\title{
Harmonic Flow for Histogram Matching
}

\author{
Thomas Batard and Marcelo Bertalmío * \\ Department of Information and Communication Technologies \\ University Pompeu Fabra, Barcelona, Spain \\ \{thomas.batard, marcelo. bertalmio\}@upf .edu
}

\begin{abstract}
We present a method to perform histogram matching between two color images based on the concept of harmonic mapping between Riemannian manifolds. The key idea is to associate the histogram of a color image to a Riemannian manifold. In this context, the energy of the matching between the two images is measured by the Dirichlet energy of the mapping between the Riemannian manifolds. Then, we assimilate optimal matchings to critical points of the Dirichlet energy. Such points are called harmonic maps. As there is no explicit expression for harmonic maps in general, we use a gradient descent flow with boundary condition to reach them, that we call harmonic flow. We present an application to color transfer, however many others applications can be envisaged using this general framework.
\end{abstract}

Keywords: Histogram matching; Variational method; Riemannian geometry; Dirichlet energy; Color transfer

\section{Introduction}

As mentioned by Ling and Okada [14], histogram-based local descriptors are used in many computer vision tasks, such as shape matching, image retrieval, texture analysis, color analysis, 3D object recognition. See also [14] for references. In order to compare histograms, it is necessary to match them first in many cases because they are not aligned (due to shape deformation, lighting conditions change, noise, etc). In [14], they make use of the Earth Moving's Distance (EMD) to compare histograms, a method that involves a histogram matching step.

In this paper, we provide a new distance for comparing histograms based on the Dirichlet energy. As the EMD, it is based on optimal matching between two sets. We present a straightforward application of histogram matching to color transfer. However, we would like to point out that this general framework can be applied to the computer vision tasks mentioned above up to small adjustments.

\footnotetext{
* This work was supported by European Research Council, Starting Grant ref. 306337. The second author acknowledges partial support by Spanish grants AACC, ref. TIN2011-15954-E, and Plan Nacional, ref. TIN2012-38112.
} 


\subsection{Color transfer}

In cinema post-production and professional photography, it is sometimes required to adjust the colors of a rendered image with respect to the colors of an other image. Such a technique is called color transfer. In the color transfer literature, the rendered image is usually called source image, and the other one is called reference image. Color transfer methods can be used for pure artistic purpose, or to solve technical issues. There exist plenty of techniques to perform color transfer between two images, but they all follow the same general idea: produce an image that shares the details of the source image and the color palette of the reference image. We can distinguish three classes of color transfer methods.

Global approaches The global approaches only take into account the whole histogram information of the two images, i.e. global information, to perform the transfer of colors. As a consequence, these approaches modify the pixels of the source image of same colors in the same way, regardless of their location in the image and the values of the neighboring pixels.

The seminal work of Reinhard et al. [21] for color transfer is based on statistical matching: the color histograms of the two images are first expressed in an uncorrelated space $l \alpha \beta$, then the histogram of the source image is modified in such a way that the mean and variance along each one of the three axis match with the one of the reference image. A similar approach has been adopted by Kotera [13] where the statistical correspondence is done through the Principal Component Analysis of the color histograms expressed in the $R G B$ space. Let us also mention the approach of Pitié et al. [18] who make use of probability distributions transfer in color spaces for performing color transfer between images.

Because these methods are global, the details of the source image as well as the color distribution of the reference image might not be preserved correctly.

Local and mixed approaches The local approaches take into account the location of the colors in the images and the values of the neighboring pixels, but not the global information. Local approaches are mainly used when the two images share some content (see e.g. [3],[4],[11]).

The mixed approaches both take into account global, i.e. histogram, information as well as local information to perform color transfer. They include most of the variational formulations of the color transfer literature (see e.g. $[5],[9],[17],[19],[20],[25])$.

In this paper, we are interested in the general case, i.e. the two images are not required to share any content. The global and mixed approaches aforementioned apply to any images, unlike the local approaches. Even if the mixed approaches provide better results than global approaches in general, it is still possible to find examples where the methods fail, due the creation of artefacts, a lack of preservation of scene details and/or color palette, an unnatural look, etc.

Hence, we claim that there is still room for improvment in color transfer. 


\subsection{Our contribution}

In this paper, we present a new formulation for histogram matching between two images (or patches) as the problem of finding an equilibrium state for the energy of the mapping of the color histogram of the source image onto the one of the reference image. More precisely, we make use of the theory of harmonic mapping between Riemannian manifolds.

Harmonic maps are critical points of the Dirichlet energy, and generalize the concepts of geodesic curves and minimal surfaces. Harmonic mapping between Riemannian manifolds has been studied for a while (see [8] and the references therein). Whereas there exists no general theory providing an expression of harmonic maps, the problem of the existence of harmonic maps has been widely investigated and different approaches have been adopted. In this paper, we follow the approach of deformation by heat flow of Eells and Sampson [7], that we call harmonic flow. The harmonic flow is nothing but the continuous formulation of the gradient descent associated to the Dirichlet energy. In particular they showed that, under some assumption on the curvature of the target manifold, the heat flow converges to a harmonic map.

The Dirichlet energy and its minimization problem were introduced for image processing by Sochen et al. [22] with an application to color image denoising. Apart from image processing, they have been applied (in a simplified form) for computer vision tasks like optimal flow estimation [2] and shape analysis [1]. In our approach of histogram matching from harmonic mapping, the Riemannian manifolds considered are related to the histograms of the images, and the local variations of the histograms are encoded into the Riemannian metrics. The choice of the Riemannian manifolds involved in our variational formulation makes the proposed color transfer method be a global approach. However, we would like to point out that others choices of Riemannian manifolds would lead to mixed or local approaches.

\section{Harmonic flow between Riemannian manifolds}

\subsection{Harmonic mapping between Riemannian manifolds}

Let $(M, g)$ and $(N, h)$ be two smooth Riemannian manifolds. The Dirichlet energy $E$ of a map $\Phi:(M, g) \longrightarrow(N, h)$ is defined by

$$
E(\Phi):=\int_{M}|d \Phi|_{g^{-1} \otimes h}^{2} d M=\int_{M} h_{\alpha \beta} \frac{\partial \Phi^{\alpha}}{\partial x^{i}} \frac{\partial \Phi^{\beta}}{\partial x^{j}} g^{i j} d M
$$

where $d \Phi$ is the differential of $\Phi$. The notation ||$_{g^{-1} \otimes h}$ stands for the norm with respect to the metric $g^{-1} \otimes h$. See [8] for more details.

The critical points of the energy (1) are called harmonic maps. They are the solutions of the system

$$
\tau(\Phi):=\Delta_{g} \Phi^{i}+\Gamma_{j k}^{i} \partial_{\mu} \Phi^{j} \partial_{\nu} \Phi^{k} g^{\mu \nu}=0, \quad i=1, \cdots, \operatorname{dim} N
$$


where $\Delta_{g}$ stands for the Laplace-Beltrami operator on $(M, g)$ and $\Gamma_{j k}^{i}$ are the symbols of the Levi-Civita connection on $(N, h)$.

The term $\tau(\Phi)$ is called the tension field of the map $\Phi$. There exists a more compact formulation for $\tau(\Phi)$ :

$$
\tau(\Phi)=\operatorname{div}(d \Phi)
$$

where div is the adjoint operator of the Levi-Civita covariant derivative $\nabla$ on the vector bundle $\Phi^{-1} T N$ over $M$, i.e. it satisfies

$$
\int_{M}\langle\nabla \varphi, \psi\rangle_{g^{-1} \otimes h} d M=\int_{M}\langle\varphi, \operatorname{div} \psi\rangle_{h} d M
$$

$\forall \varphi \in \Gamma\left(\Phi^{-1} T N\right)$ and $\psi \in \Gamma\left(T^{*} M \otimes \Phi^{-1} T N\right)$ with compact support.

Harmonic maps appear in well-known contexts:

- If $\operatorname{dim} M=1$, then the Dirichlet energy measures the length of curves in the Riemannian manifold $(N, h)$ and the harmonic maps are the geodesics of $(N, h)$.

- If $\operatorname{dim} M=2$ and $N=\mathbb{R}^{3}$, the Dirichlet energy measures the area of surfaces embedded in $\mathbb{R}^{3}$. The harmonic maps are then the minimal surfaces.

\subsection{On the harmonic flow}

In a very complete report, Eells and Lemaire [8] mentioned that there is no general theory to construct solutions of the system (2), i.e. harmonic maps between Riemannian manifolds. However, the problem of the existence of non trivial solutions has been studied for a while and is still under investigation. The classic approach is formulated as follows: "Let $\Phi_{0}: M \longrightarrow N$ be a map of Riemannian manifolds. Can $\Phi_{0}$ be deformed into a harmonic map $\Phi: M \longrightarrow N$ ?" There are different approaches to attack this problem:

- The direct method of variational theory (lower semicontinuity of the Dirichlet energy on compact subsets on the space $\mathcal{C}(M, N)$ of maps, in a suitable weak topology). This technique works well for $\operatorname{dim} M=1,2$ (see Morrey [15]), but not for $\operatorname{dim} M \geq 3$ in general.

- Deformation by heat flow: these are the approaches of Eells and Sampson [7], Hamilton [12], to show that the answer is "yes" if the sectional curvature of $N$ is nonpositive.

- Morse theory in manifolds of maps: that was used for instance by Palais [16] to prove the existence of geodesics joining two points.

In this paper, we focus on the technique of deformation by heat flow, that we call harmonic flow. 
From now on, we assume that $M$ and $N$ are compact manifolds, and $M$ has a boundary $\partial M$. Let $\psi: \partial M \longrightarrow N$ and $\Phi_{0}: M \longrightarrow N$ such that $\Phi_{0 \mid \partial M}=\psi$. We consider the harmonic flow with Dirichlet boundary conditions

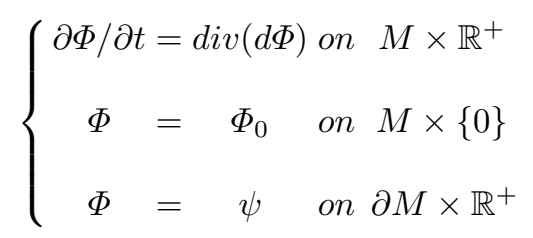

Then, there is a solution for the problem (4) defined for a short time $\left[0, t_{1}[\right.$. Moreover, $E(\Phi)$ is a strictly decreasing function of $t$, except at those values of $t$ for which $\tau(\Phi)=0$.

We have the following theorem due to Hamilton.

Theorem 1. If the Riemannian sectional curvature of $N$ is nonpositive then the solution $\Phi$ of the system (4) is globally defined and converges towards a harmonic map.

We refer to Hamilton [12] for more details about harmonic maps of manifolds with boundary.

\section{Harmonic flow for (color) histogram matching}

\subsection{From color image to Riemannian manifold}

Given an image $I: \Omega \subset \mathbb{R}^{2} \longrightarrow R G B$, the so-called tensor of structure of $I$ is the $2 \times 2$ matrix field

$$
\left(\begin{array}{cc}
\sum_{k=1}^{3}\left(I_{x_{1}}^{k}\right)^{2} & \sum_{k=1}^{3} I_{x_{1}}^{k} I_{x_{2}}^{k} \\
\sum_{k=1}^{3} I_{x_{1}}^{k} I_{x_{2}}^{k} & \sum_{k=1}^{3}\left(I_{x_{2}}^{k}\right)^{2}
\end{array}\right)
$$

The eigenvalues and eigenvectors fields of (5) provide information about the local behaviour of the image. Indeed, the eigenvalues measure the highest and lowest directional variations of $I$ and the corresponding eigenvectors give the directions of these optimal variations. The tensor of structure has been widely used for color image processing tasks (see e.g. [6] for segmentation, and [23],[24] for denoising/regularization purposes).

It turns out that the tensor of structure (5) is quite close to a Riemannian metric. Indeed, if we denote by $\psi$ the graph of the image $I$, i.e. the map

$$
\psi:\left(x_{1}, x_{2}\right) \longmapsto\left(x_{1}, x_{2}, I^{1}\left(x_{1}, x_{2}\right), I^{2}\left(x_{1}, x_{2}\right), I^{3}\left(x_{1}, x_{2}\right)\right),
$$

then $\psi$ determines the embedding of the manifold $\Omega$ into $\mathbb{R}^{5}$, and the Euclidean metric on $\mathbb{R}^{5}$ induces a Riemannian metric $g$ on $\Omega$ of the form

$$
g=\left(\begin{array}{cc}
1+\sum_{k=1}^{3}\left(I_{x_{1}}^{k}\right)^{2} & \sum_{k=1}^{3} I_{x_{1}}^{k} I_{x_{2}}^{k} \\
\sum_{k=1}^{3} I_{x_{1}}^{k} I_{x_{2}}^{k} & 1+\sum_{k=1}^{3}\left(I_{x_{2}}^{k}\right)^{2}
\end{array}\right)
$$


in the frame $\left(\partial / \partial x_{1}, \partial / \partial x_{2}\right)$. The couple $(\Omega, g)$ forms a compact Riemannian manifold of dimension 2 .

In [22], an anisotropic diffusion based on the Laplace-Beltrami operator $\Delta_{g}$ associated to the metric (6) is performed for color image denoising/regularization purposes.

In the following paragraph, we extend this construction to manifolds of dimension 3 in order to encode the local behavior of color images histograms into Riemannian metrics.

\subsection{Extension to color histogram}

Let $I: \Omega \subset \mathbb{R}^{2} \longrightarrow R G B$ be a color image. We denote by $H_{I}$ the function that maps any color $(r, g, b)$ to the number of pixels of color $(r, g, b)$ in $I$. Let $\psi$ be the graph of the function $\beta H_{I}, \beta \geq 0$, i.e.

$$
\psi:(r, g, b) \longmapsto\left(r, g, b, \beta H_{I}(r, g, b)\right)
$$

The map $\psi$ determines the embedding of the compact manifold $R G B$ of dimension 3 into $R G B \times \mathbb{R}$. Then the Euclidean metric on $R G B \times \mathbb{R}$ induces a Riemannian metric $h_{\beta}$ on $R G B$ of the form

$$
h_{\beta}=\left(\begin{array}{ccc}
1+\beta^{2}\left(\frac{\partial H_{I}}{\partial r}\right)^{2} & \beta^{2} \frac{\partial H_{I}}{\partial r} \frac{\partial H_{I}}{\partial g} & \beta^{2} \frac{\partial H_{I}}{\partial r} \frac{\partial H_{I}}{\partial b} \\
\beta^{2} \frac{\partial H_{I}}{\partial r} \frac{\partial H_{I}}{\partial g} & 1+\beta^{2}\left(\frac{\partial H_{I}}{\partial g}\right)^{2} & \beta^{2} \frac{\partial H_{I}}{\partial g} \frac{\partial H_{I}}{\partial b} \\
\beta^{2} \frac{\partial H_{I}}{\partial r} \frac{\partial H_{I}}{\partial b} & \beta^{2} \frac{\partial H_{I}}{\partial g} \frac{\partial H_{I}}{\partial b} & 1+\beta^{2}\left(\frac{\partial H_{I}}{\partial b}\right)^{2}
\end{array}\right)
$$

in the frame $(\partial / \partial r, \partial / \partial g, \partial / \partial b)$. The couple $\left(R G B, h_{\beta}\right)$ forms a compact Riemannian manifold of dimension 3 .

Remark 1. Notice that such a construction can be done for images with any number of channels.

Assuming that the manifolds $(M, g)$ and $(N, h)$ are respectively of the form $\left(R G B, h_{\beta_{1}}\right)$ and $\left(R G B, h_{\beta_{2}}\right)$, we deduce that the harmonic flow (4) converges towards a harmonic map if the sectional curvature of the Riemannian manifold $\left(R G B, h_{\beta_{2}}\right)$ is nonpositive. Let us distinguish two cases:

1. The case $\beta_{2}=\mathbf{0}$.

The Riemannian metric $h_{\beta_{2}}$ is then Euclidean (see formula (7)), the sectional curvature of $\left(R G B, h_{\beta_{2}}\right)$ is zero, and theorem 1 applies. In this case we obtain the heat flow of the Laplace-Beltrami operator $\Delta_{h_{\beta_{1}}}$ since the terms $\Gamma_{i j}^{k}$ in the tension field (2) vanish. 
2. The case $\beta_{2}>0$.

In this case, we do not know if $\left(R G B, h_{\beta_{2}}\right)$ has a nonpositive sectional curvature or not. However, we do have a necessary condition for the sectional curvature to be nonpositive. Indeed, it is mentioned in [10] that Riemannian manifolds that are hypersurfaces of $\mathbb{R}^{n}, n \geq 4$, can not have strictly negative sectional curvature. Hence, because the manifolds $\left(R G B, h_{\beta_{2}}\right)$ are hypersurfaces of $\mathbb{R}^{4}$ (see construction in Sect. 2.2), we deduce that a necessary condition is that the sectional curvature of $\left(R G B, h_{\beta_{2}}\right)$ vanishes at least once. If not, then the theorem 1 does not apply.

\subsection{Implementation}

We adopt a gradient descent approach to approximate the harmonic flow (4) for color histogram matching. The algorithm we propose is the following

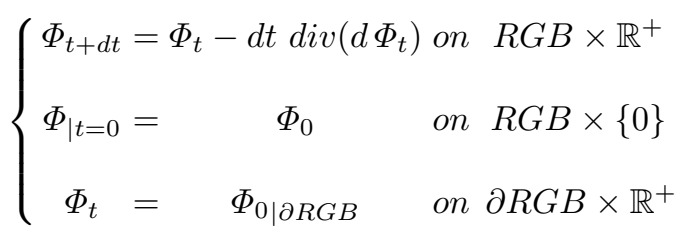

We stop the algorithm (8) at $t=T$ when the Dirichlet energy $E\left(\Phi_{t}\right)$ stops decreasing. We would like to remind that we do not have guarantee that the system (8) is globally defined if the sectional curvature of $\left(R G B, h_{\beta_{2}}\right)$ is not nonpositive. Hence, the map $\Phi_{T}$ might not be harmonic.

The main task is to compute a discrete approximation of the operator div. To perform this, our approach is based on a generalization of the Euclidean case. The classic approach to compute a discrete approximation of the Euclidean divergence div : $\Gamma\left(T^{*} \Omega\right) \longrightarrow C^{\infty}(\Omega)$ is to make use of its adjoint operator property. Indeed, by definition, we have the equality

$$
\int_{\Omega} \operatorname{div} \eta \varphi d \Omega=\int_{\Omega}\langle\eta, d \varphi\rangle d \Omega
$$

for $\varphi \in C^{\infty}(\Omega)$ and $\eta \in \Gamma\left(T^{*} \Omega\right)$ with compact support. Then, using forward differences for discretizing $d \varphi$ implies that div $\eta$ must be discretized using backward differences. We extend this approach to the operator div: $\Gamma\left(T^{*} M \otimes\right.$ $\left.\Phi^{-1} T N\right) \longrightarrow \Gamma\left(\Phi^{-1} T N\right)$ using the discrete version of the formula (3). The explicit expression of the discrete divergence operator is given in the Appendix.

\section{An application of histogram matching to color transfer}

\subsection{From harmonic mapping between color histograms to color transfer}

The method we propose for performing color transfer from a reference image $I_{r}$ to a source image $I_{s}$ is composed of two steps. 
1. We construct Riemannian manifolds $\left(R G B, h_{\beta_{s}}\right)$ and $\left(R G B, h_{\beta_{r}}\right)$ associated respectively to the images $I_{s}$ and $I_{r}$, for some values $\beta_{s}, \beta_{r}$. Then, we construct a harmonic map from $\left(R G B, h_{\beta_{s}}\right)$ to $\left(R G B, h_{\beta_{r}}\right)$. From Sect. 2.1, this is a function $\Phi_{\text {crit }}$ satisfying

$$
\operatorname{div}\left(d \Phi_{\text {crit }}\right)=0
$$

where div is the adjoint operator of the Levi-Civita covariant derivative on the bundle $\Phi_{c r i t}^{-1}\left(T R G B, h_{\beta_{r}}\right)$ over the Riemannian manifold $\left(R G B, h_{\beta_{s}}\right)$.

2. Once we get a harmonic map $\Phi_{c r i t}$, we perform color transfer from $I_{r}$ to $I_{s}$ by assigning the color $\Phi_{\text {crit }}\left(c_{1}, c_{2}, c_{3}\right)$ to the pixels of $I_{s}$ of color $\left(c_{1}, c_{2}, c_{3}\right)$, for any color $\left(c_{1}, c_{2}, c_{3}\right)$ in $I_{s}$. In other words, the output of the method is the color image Res: $\Omega \longrightarrow R G B$ defined by

$$
\operatorname{Res}(x)=\Phi_{c r i t} \circ I_{s}(x)
$$

Remark 2. The color transfer is said optimal because $\Phi_{\text {crit }}$ is harmonic. Actually, the construction in step 2 does not require the map $\Phi_{c r i t}$ to be harmonic, and any map $\Phi: R G B \longrightarrow R G B$ can generate a color transfer from $I_{r}$ to $I_{s}$.

\subsection{On the construction of the initial condition}

Because of our choice to construct harmonic maps from the heat deformation (4), our color transfer method is strongly dependent of the initial condition $\Phi_{0}: R G B \longrightarrow R G B$. Our approach consists in taking the map $\Phi_{0}$ related to any color transfer method of the literature and show that the harmonic flow improves the quality of the color transfer.

The output Res of any color transfer method might be written as follows

$$
\operatorname{Res}(x)=K \circ I_{s}(x)
$$

for some operator $K: \mathcal{C}_{I_{s}} \longrightarrow R G B$ defined on the color palette $\mathcal{C}_{I_{s}}$ of $I_{s}$.

Then, a straightforward way to construct an initial condition $\Phi_{0}$ from any color transfer method is the following. We define $\Phi_{0}: R G B \longrightarrow R G B$ as

$$
\Phi_{0}\left(c_{1}, c_{2}, c_{3}\right)=\left\{\begin{array}{cl}
K\left(c_{1}, c_{2}, c_{3}\right) & \text { if } I_{s}(x)=\left(c_{1}, c_{2}, c_{3}\right) \text { for some pixel } x \\
\left(c_{1}, c_{2}, c_{3}\right) & \text { otherwise }
\end{array}\right.
$$

\subsection{Experiments}

On Fig. 1, we show results of the color transfer method detailed above. The source images are in the top row, the reference images in the bottom row. We apply the harmonic flow described above, for some parameters $\beta_{s}, \beta_{r}, d t$, on the 
images of third row, and the results are shown in the second column.

More precisely, the initial conditions $\Phi_{0}$ are constructed following the method (10) applied to the algorithm of Reinhard et al. [21] (left column) and Kotera [13] (right column). The parameters of our algorithm are: $\beta_{s}=0, \beta_{r}=0.07$, $d t=0.001$ (left), and $\beta_{s}=0, \beta_{r}=0.001, d t=0.001$ (right) . In both cases, we stopped the algorithm (8) at $t=T$ when the Dirichlet energy $E\left(\Phi_{t}\right)$ stopped decreasing. As $\beta_{r}>0$ in these experiments, we do not have the guarantee that $\Phi_{T}$ is a harmonic map.

We see that our results are coherent with what we expect from a color transfer method: the output image provides fidelity with respect to both scene details of the source image and colors of the reference image. We observe that our method makes the color transfers be more realistic than the initial conditions. Indeed, under a right choice for the parameters of the 'flowers' images color transfer, we can see that our method removes almost completely the violet that appears on the leaves in the initial condition. However, as the color transfer method presented here is global, the violet reduces on the flowers too. Regarding the 'landscapes' images color transfers, we see that the initial condition tends to make the details in the clouds of the source image disappear. Applying the harmonic flow under a right choice for the parameters, allows us to recover a part of these details.

\section{Conclusion}

We have presented a new method for (color) histogram matching between two images, dealing with the Dirichlet energy of the mapping between Riemannian manifolds. In this context, we relate histograms of images with Riemannian manifolds, the local variations of the histograms being encoded by Riemannian metrics. We have adopted a deformation by heat flow, that we called harmonic flow, in order to construct harmonic maps. As this stage, we are not able to guarantee that the harmonic flow converges in any case toward a harmonic map. Further work will then be devoted to establish more theoretical results about the heat flow approach as well as investigate other approaches to construct harmonic maps.

We have presented an application to color transfer. The manifolds considered in this paper only encode histogram information of images, i.e. global information, meaning that color transfers modify pixels of same color in the same way, without taking into account their location or the values of the neighboring pixels. The examples presented have revealed the limits of such a global approach. However, the general framework introduced in this paper allows us to construct mixed and local approaches for color transfer by the construction of Riemannian manifolds that take into account local information of images. This is the subject of current research.

Finally, applications to computer vision can be envisaged from the construction of histogram-based local descriptors based on the Dirichlet energy and harmonic mapping. 


\section{Appendix. Computation of the heat flow}

For the purpose of application to color histogram matching, $(M, g)$ and $(N, h)$ are of dimension 3. Let $\varphi \in C^{\infty}(M, N)$ and $\left(e_{1}, e_{2}, e_{3}\right)$ be a frame field of the tangent bundle $(T N, h)$. Using forward derivatives for discretizing the derivatives of the components $\varphi^{j}, j=1,2,3$, of $\varphi$, we obtain

$$
\begin{gathered}
d \varphi=\sum_{i, j=1}^{3} \frac{\partial \varphi^{j}}{\partial x_{i}} d x_{i} \otimes\left(e_{j} \circ \Phi\right) \quad \text { where } \quad \frac{\partial \varphi^{j}}{\partial x_{i}}(x)= \\
{\left[\varphi^{j}\left(x_{1}+\delta_{1}(i)\left(1-\delta_{255}\left(x_{1}\right)\right), x_{2}+\delta_{2}(i)\left(1-\delta_{255}\left(x_{2}\right), x_{3}+\delta_{3}(i)\left(1-\delta_{255}\left(x_{3}\right)\right)\right)-\varphi^{j}(x)\right]\right.}
\end{gathered}
$$

Let $\eta=\sum_{i, j=1}^{3} \eta^{i j} d x_{i} \otimes\left(e_{j} \circ \Phi\right) \in \Gamma\left(T^{*} M \otimes \Phi^{-1} T N\right)$. From the discretization of the formula (3) and the discrete formula for the covariant derivative $\nabla$ induced by formula (11), we obtain

$$
\begin{gathered}
\operatorname{div}(\eta)=\sum_{i, j=1}^{3} A_{j}\left(h^{i j} \circ \Phi\right)\left(e_{i} \circ \Phi\right) \quad \text { where } \quad\left(A_{j}\right)(x)= \\
\sum_{a, b, c=1}^{3}\left[\left(g^{a b}\left(h_{c j} \circ \Phi\right) \eta^{c b}\right)\left(x_{1}-\delta_{1}(a), x_{2}-\delta_{2}(a), x_{3}-\delta_{3}(a)\right)-\left(g^{a b}\left(h_{c j} \circ \Phi\right) \eta^{c b}\right)(x)\right] \\
+\sum_{\alpha, \beta, \mu, \nu=1}^{3} \eta^{\alpha \beta}\left(g^{\mu \alpha}\left(h_{\beta \nu} \circ \Phi\right)\left(\Gamma_{\mu \nu}^{j} \circ \Phi\right)\right)(x) \quad \text { if } \quad x \notin \partial M
\end{gathered}
$$

where $\Gamma_{\mu \nu}^{j}$ are the symbols of the Levi-Civita connection on $(T N, h)$.

In order that the heat flow satisfies the Dirichlet boundary conditions, the quantity div $\circ d$ must vanish on $\partial M$. On the other hand, because the Dirichlet energy depends also on the values of $d$ at the boundary, we should not impose that $d \equiv 0$ at the boundary. Hence, we make the choice of imposing $\operatorname{div}_{\mid \partial M} \equiv 0$ in order to guarantee that the Dirichlet boundary condition is satisfied.

Finally, computing the differential $d \Phi_{t}$ using formula (11) and applying formula (12) for $\eta:=d \Phi_{t}$ gives the expression of the algorithm (8).

\section{References}

1. Aflalo, Y., Kimmel, R., Zibulevsky, M.: Conformal Mapping with as Uniform as Possible Conformal Factor. SIAM J. of Imaging Sciences, 6(1), pp. 78-101 (2013)

2. Ben-Ari, R., Sochen, N.: A Geometric Framework and a New Criterion in Optical Flow Modeling. J. Mathematical Imaging and Vision, 33(2), pp. 178-194 (2009)

3. Bertalmío, M., Levine, S.: Variational Approach for the Fusion of Exposure Bracketed Pairs. IEEE Trans. Image Processing, 22(2), pp. 712-723 (2013)

4. Bertalmío, M., Levine, S.: Color Matching for Stereoscopic Cinema. Proc. of MIRAGE 2013, 6 (2013)

5. Delon, J.: Midway Image Equalization. J. Mathematical Imaging and Vision 21(2), pp. 119-134 (2004) 
6. Di Zenzo, S.: A Note on the Gradient of a Multi-Image. Computer Vision, Graphics and Image Processing, 33(1), pp. 116-125 (1986)

7. Eells, J., Sampson, J.H.: Harmonic Mappings of Riemannian Manifolds. American Journal of Mathematics, 86(1), pp. 109-160 (1964)

8. Eells, J., Lemaire, L: A Report on Harmonic Maps. Bull. London Math. Soc., 10, pp. 1-68 (1978)

9. Ferradans, S., Papadakis, N., Rabin, J., Peyré, G., Aujol, J.-F.: Regularized Discrete Optimal Transport. Proc. 4th Int. Conf. SSVM, LNCS 7893, pp. 428-439 (Kuijper, A. et al. Eds) (2013)

10. Gallot, S., Hulin, D., LaFontaine, J.: Riemannian Geometry. Springer (2004)

11. HaCohen, Y., Shechtman, E., Goldman, D.B., Lischinski, D.: Non-Rigid Dense Correspondence with Applications for Image Enhancement. ACM Trans. Graph., 30(4), Art. 70 (2011)

12. Hamilton, R.S.: Harmonic Maps of Manifolds with Boundary. Lecture in Mathematics, 471, Springer (1975)

13. Kotera, H.: A Scene-Referred Color Transfer for Pleasant Imaging on Display. IEEE Int. Conf. Image Processing, 2, pp. 5-8 (2012)

14. Ling, H., Okada, K.: An Efficient Earth Mover's Distance Algorithm for Robust Histogram Comparison. IEEE Trans. Pattern Analysis and Machine Intelligence, 29(5), pp. 840-853 (2007)

15. Morrey, C. B.: The Problem of Plateau on a Riemannian Manifold. Annals of Mathematics, 49, pp. 807-851 (1948)

16. Palais, R.S.: Morse Theory on Hilbert Manifolds. Topology, 2, pp. 299-340 (1963)

17. Papadakis, N., Provenzi, E., Caselles, V.: A Variational Model for Histogram Transfer of Color Images. IEEE Trans. Image Processing, 20(6), pp.1682-1695 (2011)

18. Pitié, F., Kokaram, A., Dahyot A.: Automated Color Grading using Colour Distribution Transfer. Computer Vision and Image Understanding, 107(1), pp.123-137 (2007)

19. Pitié, F., Kokaram, A.: The Linear Monge-Kantorovitch Colour Mapping for Example-based Colour Transfer. Proc. IEEE Eur. Conf. Vis. Media Prod., pp.1-9 (2007)

20. Rabin, J., Peyré, G.: Wasserstein Regularization of Imaging Problem. Proc. of 17th IEEE Int. Conf. Image Processing ICIP, pp. 1541-1544 (2011)

21. Reinhard, E., Ashikhmin, M., Gooch, B., Shirley, P.: Color Transfer between Images. IEEE Computer Graphics and Applications, 21(5), pp. 34-41 (2001)

22. Sochen, N., Kimmel, R., Malladi, R.: A General Framework for Low Level Vision. IEEE Trans. Image Processing, 7(3), pp. 310-318 (1998)

23. Tschumperlé, D., Deriche, R.: Vector-Valued Image Regularization with PDEs: A Common Framework for Different Applications. IEEE Trans. Pattern Anal. Mach. Intell., 27(4), pp. 506-517 (2005)

24. Weickert, J.: Anisotropic Diffusion in Image Processing. Teubner, Stuttgart (1998)

25. Xiao, X., Ma, L.: Gradient-Preserving Color Transfer. Computer Graphics Forum, 28(7), pp. 1879-1886 (2009) 

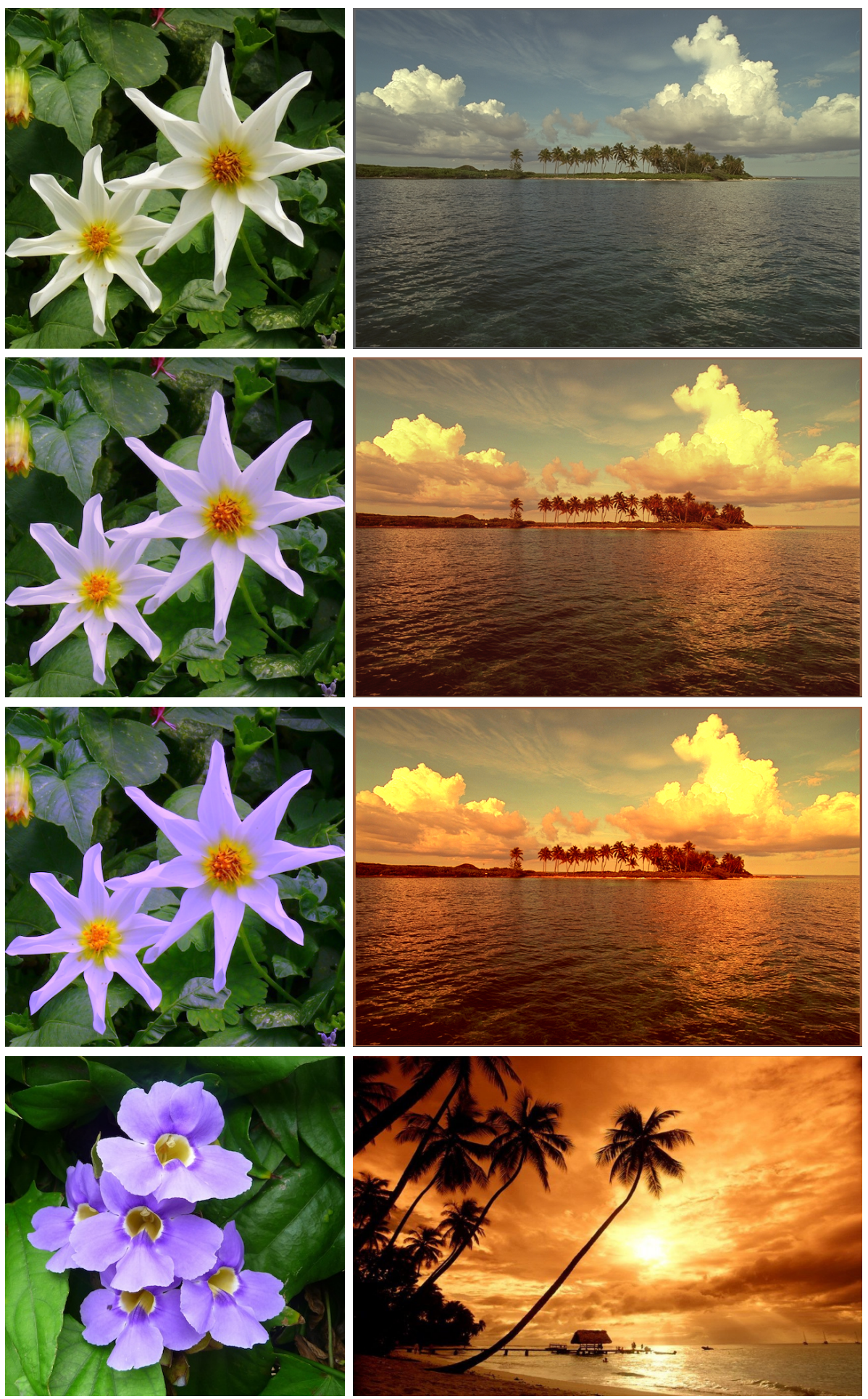

Fig. 1. Harmonic flow for color transfer. Top row: source images; bottom row: reference images; third row: color transfer methods of Reinhard et al. [21] (left), Kotera [13](right); second row: results of harmonic flows applied to the initial conditions. 